\title{
A pheochromocytoma case diagnosed as adrenal incidentaloma
}

\author{
Doğuş Vurallı ${ }^{1}$, Nurgün Kandemir ${ }^{1}$, Graeme Clark$^{2}$, Diclehan Orhan ${ }^{3}$, Ayfer Alikaşifoğlu ${ }^{1}$, \\ Nazlı Gönç ${ }^{1}$, Saniye Ekinci ${ }^{4}$, Alev Özön ${ }^{1}$ \\ Division of ${ }^{1}$ Pediatric Endocrinology Departments of Pediatrics, ${ }^{3}$ Pathology, and ${ }^{4}$ Pediatric Surgery, Hacettepe University \\ Faculty of Medicine, Ankara, Turkey; ${ }^{2}$ University of Cambridge and NIHR Cambridge Biomedical Research Center, \\ Department of Medical Genetics, Cambridge, United Kingdom. E-mail: dvuralli@hotmail.com \\ Received: 3rd November 2016, Revised: 24th January 2017, Accepted: 16th February 2017
}

SUMMARY: Vurallı D, Kandemir N, Clark G, Orhan D, Alikaşifoğlu A, Gönç N, Ekinci S, Özön A. A pheochromocytoma case diagnosed as adrenal incidentaloma. Turk J Pediatr 2017; 59: 200-206.

There are two problems that needs to be addressed in cases of an adrenal incidentaloma. The first is to decide whether the adrenal mass is benign or malignant, and the second is to determine whether the mass is hormonally active or not. A 17-year-old male was admitted with the complaint of progressive weight gain. Abdominal ultrasonography was performed for elevation in transaminases which revealed a hypoechoic mass located in the left adrenal gland. Hormonal investigations revealed an increase in fractionated catecholamine and metanephrine levels in 24-hour urine. Surgery was performed and pathological examination was in accordance with pheochromocytoma. Mutation analysis was carried out. This is a rare case of pheochromocytoma presenting as adrenal incidentaloma during adolescence. In view of this case, we review the approach to incidentally discovered adrenal masses and the approach to pheochromocytoma. A mutation analysis should be performed on all cases with pheochromocytoma that are diagnosed below age 20 .

Key words: adrenal glands, adrenal gland neoplasms, adrenal incidentaloma, pheochromocytoma.

Adrenal masses larger than $1 \mathrm{~cm}$ determined incidentally during radiological examinations or laparotomy with no suspicion for a specific adrenal pathology, are defined as adrenal incidentalomas. Refinement of radiological techniques and their widespread use led to an increase in such cases. In various series, prevalence of incidentalomas was reported to be $1-8.7 \%^{1-3}$. The prevalence increases with age, from $0.2 \%$ during childhood and adolescence, up to $10 \%$ after the 5 th decade of life ${ }^{4}$. Etiological distribution of adrenal incidentalomas is different between clinical and surgical studies. In clinical studies, nonfunctional adenomas are the most frequent form, followed by functional adenomas and other tumors such as pheochromocytoma, carcinoma and metastatic tumors. Nonfunctional adenomas comprise $70-75 \%$ of the cases in clinical studies whereas the proportion of cortisoland aldosterone-secreting adenomas is nearly $15-20 \%$, pheochromocytoma, carcinoma, and metastatic tumors are responsible for $5-10 \%$, $5-10 \%$, and $5 \%$, respectively 4 . Prevalence of adenomas decreases to about $55-60 \%$ in surgical series, whereas the prevalence of other tumors like pheochromocytoma, carcinoma, ganglioneuroma, cysts, myelolipoma, and metastatic tumors are higher than clinical studies ${ }^{4}$. Herein we report a case with pheochromocytoma presenting as incidentaloma which is rare during childhood. The approach to adrenal tumors during childhood is reviewed.

\section{Case Report}

A 17-year-old male patient was admitted to the pediatric endocrinology outpatient clinic with the complaint of progressive weight gain over the last 4-5 years. Personal or familial history showed no peculiarities. On physical examination, body weight was $88.5 \mathrm{~kg}$ (90-97 p), height $170 \mathrm{~cm}(10-25 \mathrm{p})$, and body mass index $30.6 \mathrm{~kg} / \mathrm{m}^{2}$ (>95 p). Vitals were within 
normal limits; pulse $80 / \mathrm{min}$ and rhythmic, blood pressure $120 / 80 \mathrm{mmHg}$ ( $<90^{\text {th }}$ percentile of $130 / 81 \mathrm{mmHg}$ ). Systemic findings were also normal. Complete blood count, serum electrolytes, and renal function tests were all within normal range (NR). Liver function tests revealed elevation of transaminases: AST 118 U/L (NR: <33), ALT 115 U/L (NR: <26). Serum lipids were also elevated: LDL $183 \mathrm{mg} /$ dl (NR: 60-130), triglyceride $220 \mathrm{mg} / \mathrm{dl}$ (NR: 50-200)). Fasting blood glucose was $105 \mathrm{mg} /$ dl (NR: 70-100); however, fasting glucose and glucose tolerance were normal during an oral glucose tolerance test. Thyroid hormone levels were normal.

Elevated transaminases suggested steatohepatitis which led to an abdominal ultrasonography. This revealed grade 2 steatohepatitis as well as hepatomegaly. Significantly, it also showed a hypoechoic mass of $6.0 \times 5.1 \mathrm{~cm}$, with smooth borders in the left adrenal gland. An abdominal computerized tomography (CT) study confirmed a tumor of $7.0 \times 5.0 \times 6.7 \mathrm{~cm}$ in the left adrenal gland with cystic necrotic areas. The tumor was enhanced heterogeneously after IV contrast and there was compression of the surrounding tissues. These findings led to the diagnosis of adrenal incidentaloma. The patient was re-evaluated clinically and signs of excess cortisol such as moon face, plethora, purple striae, easy bruising, proximal muscle weakness, or hyperglycemia were absent. There was no polyuria, polydipsia or hypokalemia excluding excess aldosterone secretion clinically. Findings related to excess catecholamine secretion such as palpitations, headache, sweating episodes, paleness or flushed face, anxiety, tremors, high fever, paresthesia, diarrhea or constipation were also absent. Blood pressure measurements were within the normal range during follow-up. A more detailed examination of blood pressure using 24-hour blood pressure monitoring revealed hypertensive values. The selective alpha-1 antagonist, doxazocin, was prescribed for hypertension. The patient was also evaluated for possibility of a functional tumor (Table I). All hormones were within normal levels except urinary catecholamines and their metabolites. A two- to three-fold increase was detected in fractionated catecholamine and metanephrine levels in 24-hour urine, thus the patient was considered to have pheochromocytoma.

Since the mass was large $(>6.0 \mathrm{~cm})$ and hormonally active, surgery was planned. Preoperative preparation was carried out to prevent catecholamine crisis during surgery that may be caused by anesthesia or the surgery itself. Surgery was deferred 10 days for preoperative preparation to normalize blood pressure, heart rate, and blood volume. For the control of blood pressure, doxazocin was continued. Fluid replacement with IV normal saline was initiated two days before surgery to correct extracellular fluid contraction caused by excess catecholamine secretion. During the surgery Na-nitroprusside was used to prevent hypertensive crisis.

Left surrenalectomy was carried out and pathological analysis confirmed pheochromocytoma. The specimen was positively stained with neuron-specific enolase (NSE), chromogranin and PGP 9.5, negatively stained with leukocyte-common antigen (LCA). S-100 was positive in sustentacular cells. These findings suggested pheochromocytoma (Fig. 1). The fibrous capsule was intact and surgical borders were negative for tumor.

The case was evaluated for genetic syndromes that can be associated with pheochromocytoma such as multiple endocrine neoplasia type 2 (MEN2), Von Hippel Lindau disease, or neurofibromatosis type 1 (NF1). Serum $\mathrm{Ca}$, $\mathrm{PO}_{4}, \mathrm{ALP}, \mathrm{PTH}$, free T4, TSH and calcitonin were all within normal limits thus excluding MEN2 (Table II). Thyroid ultrasonography ruled out nodular thyroid disease. Neither mucosal neuroma nor marfanoid habitus were evident. The ophthalmological examination excluded retinal hemangioma. Magnetic resonance imaging (MRI) was negative for central nervous system tumors, such as hemangioblastoma, and renal pathology was not detected in the abdominal CT, thus Von Hippel Lindau disease was excluded. Findings typical of NF1 such as cafe au-lait spots, axillary freckling, neurofibroma, Lisch nodules and optic nerve glioma were not present.

DNA was extracted from a peripheral blood sample by standard methods for molecular analysis. The patient underwent whole exome analysis. Exome resequencing was performed in Cambridge Biomedical Research Centre as described previously ${ }^{5}$. In brief, exons were captured using the Nextera Rapid Capture Exome enrichment kit (Illumina) covering 
Table I. Laboratory Analysis to Evaluate the Etiology of the Tumor

\begin{tabular}{ll}
\hline Prediagnosis & Laboratory Tests Performed (normal range) \\
\hline Subclinical Cushing syndrome & Plasma ACTH level: $29.1 \mathrm{pg} / \mathrm{ml}(10-60)$ \\
& Serum cortisol level: $13.03 \mu \mathrm{g} / \mathrm{dl}(4.3-22.4)$ \\
& Nocturnal cortisol: $3 \mu \mathrm{g} / \mathrm{dl}(3.0-16.7)$ \\
& 24 hour urine free cortisol:40 $\mu \mathrm{g} / \mathrm{day}(11-84)$ \\
Primary hyperaldosteronism & Serum K: $4.1 . \mathrm{mEq} / \mathrm{L}(3.5-5)$ \\
& Renin: $15.7 \mathrm{pg} / \mathrm{ml}(5-33)$ \\
& Aldosterone: $80 \mathrm{pg} / \mathrm{ml}(30-313)$ \\
& DHEA-S: $209 \mu \mathrm{g} / \mathrm{dl}(108-441)$ \\
& Testosterone: $313 \mathrm{ng} / \mathrm{dl}(300-1200)$ \\
& 17-OH-Progesterone: $0.8 \mathrm{ng} / \mathrm{ml}(0-2)$ \\
& Catecholamine and its metabolites in 24 hour urine \\
& 3-Methoxytyramine: $170.95 \mu \mathrm{g} / \mathrm{day}(83-459.8)$ \\
& Metanephrine: $5.39 \mu \mathrm{g} / \mathrm{day}(42-347.7)$ \\
& Epinephrine: $18.96 \mu \mathrm{g} / \mathrm{day}(4-20)$ \\
& Vanilmandelic acid $(\mathrm{VMA}): 39.78 \mu \mathrm{g} / \mathrm{day}(1.6-7.3)$ \\
& 5-hydroxy indole acetic acid: 15.8 $\mu \mathrm{g} / \mathrm{day}(0.5-8.2)$ \\
& Norepinephrine: $7598.39 \mu \mathrm{g} / \mathrm{day}(23-105)$ \\
& Dopamine: $1491.64 \mu \mathrm{g} / \mathrm{day}(190-450)$ \\
& Normetanephrine: $4543.75 \mu \mathrm{g} / \mathrm{day}(72-659)$ \\
& Homovanilic acid $(\mathrm{HVA}): 10.8 \mu \mathrm{g} / \mathrm{day}(1.4-8.8)$ \\
& Plasma Catecholamines \\
& Norepinephrine: $1180.3 \mathrm{ng} / \mathrm{L}(70-480)$ \\
& Epinephrine: $38 \mathrm{ng} / \mathrm{L}(20-190)$ \\
\hline &
\end{tabular}

$45 \mathrm{Mb}$ of exonic sequence, and massively parallel DNA sequencing was performed on an Illumina HiSeq 4000 with 150 bp paired end reads. Depth and breadth of sequence coverage was calculated with custom scripts and the BedTools package. Single nucleotide substitutions and small insertion deletions were identified and quality filtered within the SamTools software package and in-house software tools. Variants were annotated with respect to genes and transcripts with the Annovar tool. Filtering of variants for novelty was performed by comparison to the following genomic databases; dbSNP139, 1000 Genomes project and the Exome Aggregation Consortium (ExAC) SNP calls. Initially, the analysis of the exome resequencing data focused only on known Pheochromocytoma/paraganglioma (PCC/PGL) predisposition genes. This involved filtering our exome resequencing data and analyzing on those variants present in the 16 currently reported PCC/PGL predisposing genes (RET, NF1, VHL, SDHA, SDHB, SDHC, SDHD, SDHAF2, TMEM127, PHD1, PHD2, HIF2A, FH, MAX, KIF1B, and MDH2). However, after this initial filtering, no potentially pathogenic variants were identified. The filtering was then extended to include rare variants in genes previously associated with cancer (using data from the COSMIC database (http:// cancer.sanger.ac.uk/cancergenome/projects/ cosmic). Similarly, no standout candidates were observed.

One week after the operation, fractionated catecholamine and metanephrine levels in 24hour urine returned to normal, indicating that the tumor was totally removed. The case is still under follow-up with annual evaluation of 24-hour urinary fractionated catecholamine and metanephrine as well as plasma metanephrine levels, and remains recurrence free three years after the operation.

Informed consent was received from the family.

\section{Discussion}

Adrenal incidentaloma presents two main problems to be resolved; firstly, to decide whether the adrenal tumor is benign or malignant, and the second is to determine if the mass is hormonally active or not. The dimensions of the tumor and radiological characteristics help to determine if the mass is benign or malignant. Possibility of malignancy 
increases with tumors larger than $4-6 \mathrm{~cm}^{1,6}$. CT is the ideal radiological method for visualization of the adrenal gland and CT attenuation value, expressed in Hounsfield units (HU), is important in the differential diagnosis of the mass. Adenomas are lipid rich and typically have low attenuation values of $\leq 10$ units. To discriminate between benign and malignant tumors, $10 \mathrm{HU}$ is accepted as the threshold and in previous studies sensitivity and specificity of this threshold to discriminate benign masses from malignant ones were shown to be 50 $75 \%$ and $96-100 \%$ respectively ${ }^{4}$. Fat content of malignant tumors is usually low and they generally have attenuation values higher than 20 HU. Irregular borders, heterogeneous appearance, dense contrast enhancement, invasion into the neighboring tissues, and metastatic retroperitoneal lymph nodes are important for determination of malignancy. Cases with attenuation value between 10$20 \mathrm{HU}$ on CT are suspicious for malignancy and may be further evaluated by MRI. Tissue specificity is high on MRI and adrenal-liver, adrenal-spleen, and adrenal-muscle mass signal intensity ratios can be calculated ${ }^{7}$. The mass in this case was larger than $6 \mathrm{~cm}$, with a heterogeneous appearance and it consisted of calcified and cystic necrotic areas. On CT, the adrenal mass had an attenuation value of 20 HU. Dense contrast enhancement was present suggesting hypervascularization and it did not disappear rapidly after injection with washout being $<60 \%$ after 10 minutes. These findings suggested that the mass was malignant rather than an adenoma.

The second question that needs to be addressed is whether the mass is hormonally active or not. Adrenal cortical lesions may secrete cortisol, aldosterone or androgens, whereas adrenomedullary tumors may secrete catecholamines and their metabolites. Cortisol oversecretion may lead to clinically overt or silent (subclinical) Cushing's syndrome (CS), aldosterone oversecretion leads to primary aldosteronism, and androgen oversecretion leads to virilization. Adrenomedullary tumors secreting catecholamines and their metabolites are known as pheochromocytoma. Subclinical CS can be evaluated with an overnight $1 \mathrm{mg}$ dexamethasone suppression test (DST); if postDST cortisol level is $<1.8 \mu \mathrm{g} / \mathrm{dl}$, CS is excluded, post-DST cortisol $>5 \mu \mathrm{g} / \mathrm{dl}$ may indicate subclinical CS if overt signs and symptoms of CS are lacking. When post-DST cortisol level is $1.8-5 \mu \mathrm{g} / \mathrm{dl}$, additional analyses such as low- and high-dose DSTs must be performed ${ }^{8}$. Primary hyperaldosteronism is diagnosed when plasma aldosterone concentration/plasma renin activity is $>20$, and serum aldosterone level is $>10 \mathrm{ng} / \mathrm{dl}$. Primary hyperaldosteronism should always be evaluated in hypertensive cases with an adrenal mass ${ }^{1,9}$. A suspicion for androgensecreting tumor would indicate further analysis including DHEA-S, testosterone and 17-OHprogesterone measurements.

In the diagnostic work-up of pheochromocytomas, measurement of plasma catecholamine levels have low sensitivity. The most sensitive test is plasma free metanephrine and 24-hour urinary metanephrines. The measurement of free catecholamine and its metabolites in 24-hour urine are used as a screening test $^{10}$. An elevation of at least two - three times the normal range for fractionated urine catecholamine and metanephrine levels in

Table II. Laboratory Analysis for the Genetic Syndromes Associated with Pheochromocytoma.

\begin{tabular}{ll}
\hline Genetic syndromes & Diagnostic investigation \\
\hline Multiple Endocrine Neoplasia type & Ca: $9.2 \mathrm{mg} / \mathrm{dl}(8.8-10.6)$ \\
2 (MEN2) & P: $3.73 \mathrm{mg} / \mathrm{dl}(2.7-4.9)$ \\
& ALP: $159 \mathrm{U} / \mathrm{L}(52-171)$ \\
& PTH: $30.2 \mathrm{pg} / \mathrm{ml}(12-88)$ \\
& Free T4: $12 \mathrm{pmol} / \mathrm{L}(7.86-14.41)$ \\
& TSH: $3.1 \mu \mathrm{IU} / \mathrm{ml}(0.34-5.6)$ \\
& Calcitonin: $5 \mathrm{pg} / \mathrm{ml}(0-18)$ \\
& Ophthalmological examination: Normal \\
Von Hippel Lindau disease (VHL) & Cranial MRI : Normal \\
& Abdominal CT: Normal \\
& Dermatological examination: Normal \\
Neurofibromatosis type 1 (NF1) & Ophthalmological examination: Normal \\
\end{tabular}



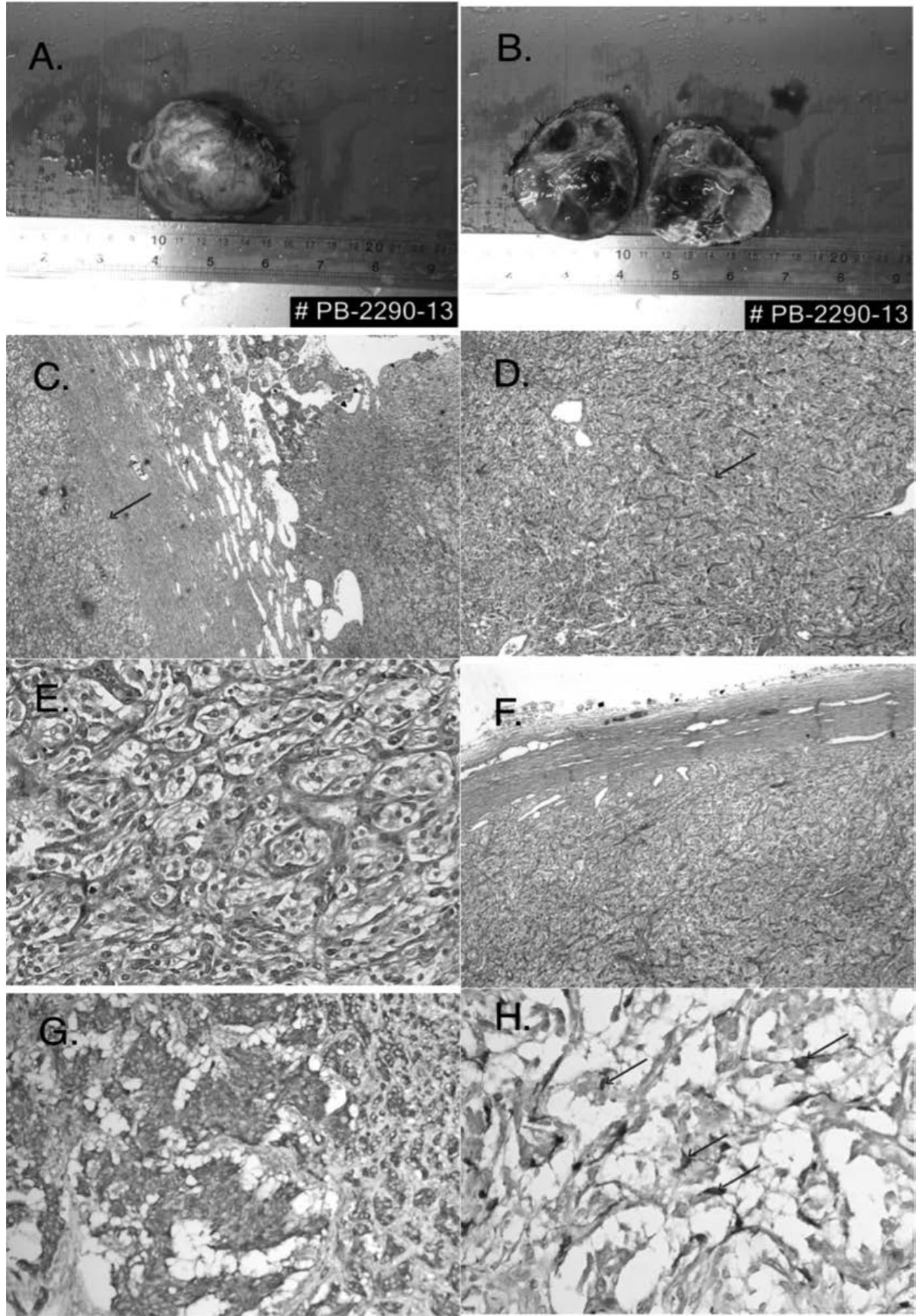

Fig. 1. Pathological examination of the tumor: A, B) Gross examinations of the tumor. C) Tumor separated from the surrenal gland by fibrous tissue (arrow) (HE, X40). D) Tumor cells forming groups surrounded by sustentacular cells (arrow) (HE, X100). E) Tumor cells having large cytoplasm and distinct nucleolus (HE, X200). F) Pheochromocytoma showing zellballen pattern surrounded by fibrous tissue (HE, X100). G) Tumor cells positively stained with chromogranin (HE, X100). H) S100 positive sustentacular cells surrounding tumor cells (arrows) (HE, X200). HE: hematoxylin-eosin. 
24-hour urine is required for a diagnosis of pheochromocytoma, which was the case in our patient.

Other diagnostic entities that should be kept in mind during evaluation of an adrenal incidentaloma, are adrenocortical carcinomas, myelolipoma, lipoma, neurofibroma, hamartoma, amyloidosis, cyst, hematoma, metastasis from other primary cancers (lung, breast, kidney, ovarian cancers, melanoma, and lymphoma $)^{3,11}$. Adrenocortical carcinomas generally have poor prognosis and $90 \%$ of them are hormonally active during childhood. Most of them secrete androgens or androgens in combination with cortisol ${ }^{12}$. One also has to keep in mind that $10-15 \%$ of incidentalomas may be bilateral. When bilateral adrenal incidentaloma is detected, metastatic disease, congenital adrenal hyperplasia, bilateral cortical adenoma, lymphoma, infection (tuberculosis, fungal), hemorrhage, ACTH-dependent CS, pheochromocytoma, amyloidosis, infiltrative diseases and ACTH-independent macronodular adrenal hyperplasia should be considered in the differential diagnosis ${ }^{13}$.

Surgery is indicated when the mass appears to be malignant, the size is larger than 4-6 $\mathrm{cm}$ or when the mass is hormonally active. In cases with pheochromocytoma, medical therapy should be initiated 7-10 days before any surgery for control of hypertension as well as correction of catecholamine induced extracellular fluid volume contraction. Alpha-adrenoreceptor blockers are used primarily to control blood pressure, with phenoxybenzamine, a longacting, irreversible, nonspecific alpha-adrenergic antagonist being the usual choice. Selective alpha-1-antagonists such as Prazosin, Doxazocin or Terazosin may also be used instead of phenoxybenzamine. Unlike phenoxybenzamine, selective antagonists do not cause reflex tachycardia, and they have a shorter duration of action allowing a more rapid dose adjustment. Since phenoxybenzamine is unavailable in Turkey, Doxazocin was used in this case. Following effective alpha-blockage, betaadrenoreceptor blockers are added to prevent tachycardia and arrhythmias. Alpha-methyltyrosine is an agent that inhibits catecholamine synthesis via inhibition of tyrosine hydroxylase. It may be used when efficient alpha- and betablockage cannot be achieved, if side-effects of blocker therapy develop or if the tumor will be intensely manipulated during the operation. Calcium channel blockers and ACE inhibitors may also be used when blood pressure is not controlled sufficiently. Normotensive patients must also receive preoperative alphaadrenoreceptor blocker therapy. However, in cases with head and neck originated parasympathetic paragangliomas that do not produce catecholamines, as well as in cases with tumors secreting only dopamine, alpha-blockers are not required ${ }^{14,15}$. Phentolamine, which is a short-acting nonselective alpha-adrenergic blocker (alpha1 and alpha2 adrenergic blocker), or alternatively Na-nitroprusside are recommended for hypertensive crisis that may develop during surgery. The second step in preoperative management is fluid replacement and it should be initiated one to three days before surgery. Excess catecholamine secretion leads to fluid contraction and hypovolemia and intravascular volume must be increased using iv normal saline. Hydration also prevents postural hypotension that is caused by alpha-blockers ${ }^{16}$.

One or two weeks after the operation, 24hour urine fractionated catecholamine and metanephrine levels should be measured in order to rule out residual tumor, multiple tumors, or metastasis. Any elevation in these hormones should be further evaluated using metaiodobenzylguanidine (MIBG) scintigraphy with ${ }^{123}$ I to detect distant metastasis. If bilateral adrenalectomy is performed, lifelong replacement with glucocorticoids and mineralocorticoids is required. If the patient is tumor free following surgery annual follow-up should be continued for recurrence or metastasis using 24-hour urine fractionated catecholamine and metanephrine as well as plasma metanephrine levels. Risk of recurrence is high in tumors localized extraadrenally or bilaterally as well as in familial cases $^{17}$.

It must be kept in mind that familial pheochromocytoma/paraganglioma syndromes are responsible for $15-20 \%$ of all cases with pheochromocytoma. These syndromes are caused by mutations in genes (SDHB, SDHC and SDHD) coding for succinate dehydrogenase subunit, part of mitochondrial complex II. Genetic screening is therefore recommended in cases with a family history of pheochromocytoma/paraganglioma, cases with bilateral pheochromocytoma, cases with paraganglioma when findings suggesting 
a genetic disorder (retinal angioma, cerebellar tumor, axillary freckling, cafe-aulait spots, medullary thyroid cancer, and hyperparathyroidism) are present, as well as all cases less than 20 years of age ${ }^{15,18,19}$. In our patient no standout candidates were observed in the analysis of exome resequencing data. The inconclusive results of genetic analysis in this case highlight pitfalls in performing exome resequencing on a single case, especially from a blood derived DNA sample alone. Although, no clear candidates were observed, several hundred rare variants remain post filtering.

In conclusion, pheochromocytoma is rare during childhood and adolescence and presentation as adrenal incidentaloma is even rarer. All cases with adrenal incidentaloma should be evaluated for pheochromocytoma and subclinical CS whereas hypertensive and/or hypokalemic cases should be evaluated for primary hyperaldosteronism as well. When the incidental mass appears to be malignant or larger in size than $4-6 \mathrm{~cm}$ or when the mass is hormonally active, surgery must be performed. Benign, nonfunctional tumors must be followed with appropriate radiological imaging and hormonal evaluation since they may rarely show malignant transformation or become hormonally active. Genetic screening is recommended in all cases with catecholaminesecreting tumors in patients below 20 years of age, even without syndromic or familial characteristics. Lifelong clinical and biochemical follow-up should be performed on a yearly basis in all cases with pheochromocytoma for an increased risk of recurrence and metastasis.

\section{REFERENCES}

1. Mansmann G, Lau J, Balk E, Rothberg M, Miyachi Y, Bornstein SR. The clinically inapparent adrenal mass: update in diagnosis and management. Endocr Rev 2004; 25: 309-340.

2. Grumbach MM, Biller BM, Braunstein GD, et al. Management of the clinically inapparent adrenal mass ("incidentaloma"). Ann Intern Med 2003; 138: 424429 .

3. Kloos RT, Gross MD, Francis IR, Korobkin M, Shapiro B. Incidentally discovered adrenal masses. Endocr Rev 1995; 16: 460-484.

4. Terzolo M, Stigliano A, Chiodini I, et al. AME position statement on adrenal incidentaloma. Eur J Endocrinol 2011; 164: 851-870.
5. Walsh DM, Shalev SA, Simpson MA, et al. Acrocallosal syndrome: identification of a novel KIF7 mutation and evidence for oligogenic inheritance. Eur J Med Genet 2013; 56: 39-42.

6. Mantero F, Terzolo M, Arnaldi G, et al. A survey on adrenal incidentaloma in Italy. Study Group on Adrenal Tumors of the Italian Society of Endocrinology. J Clin Endocrinol Metab 2000; 85: 637-644.

7. Outwater EK, Siegelman ES, Radecki PD, Piccoli CW, Mitchell DG. Distinction between benign and malignant adrenal masses: Value of T1-weighted chemical-shift MR imaging. AJR Am J Roentgenol 1995; 165: 579583.

8. Nieman LK, Biller BM, Findling JW, et al. The diagnosis of Cushing's syndrome: an Endocrine Society Clinical Practice Guideline. J Clin Endocrinol Metab 2008; 93: 1526-1540.

9. Young WF, Jr. Clinical practice. The incidentally discovered adrenal mass. N Engl J Med 2007; 356: 601-610.

10. Lenders JW, Pacak K, Walther MM, et al. Biochemical diagnosis of pheochromocytoma: which test is best? JAMA 2002; 287: 1427-1434.

11. Lam KY, Lo CY. Metastatic tumours of the adrenal glands: a 30-year experience in a teaching hospital. Clin Endocrinol (Oxf) 2002; 56: 95-101.

12. Michalkiewicz E, Sandrini R, Figueiredo B, et al Clinical and outcome characteristics of children with adrenocortical tumors: A report from the International Pediatric Adrenocortical Tumor Registry. J Clin Oncol 2004; 22: 838-845.

13. Mazzuco TL, Bourdeau I, Lacroix A. Adrenal incidentalomas and subclinical Cushing's syndrome: Diagnosis and treatment. Curr Opin Endocrinol Diabetes Obes 2009; 16: 203-210.

14. Chen H, Sippel RS, O'Dorisio MS, Vinik AI, Lloyd RV, Pacak K. The North American Neuroendocrine Tumor Society consensus guideline for the diagnosis and management of neuroendocrine tumors: Pheochromocytoma, paraganglioma, and medullary thyroid cancer. Pancreas 2010; 39: 775-783.

15. Zeiger MA, Thompson GB, Duh QY, et al. The American Association of Clinical Endocrinologists and American Association of Endocrine Surgeons medical guidelines for the management of adrenal incidentalomas. Endocr Pract 2009; 15 (Suppl 1): 1-20.

16. Hack HA. The perioperative management of children with phaeochromocytoma. Paediatr Anaesth 2000; 10: 463-476.

17. Amar L, Servais A, Gimenez-Roqueplo AP, Zinzindohoue F, Chatellier G, Plouin PF. Year of diagnosis, features at presentation, and risk of recurrence in patients with pheochromocytoma or secreting paraganglioma. J Clin Endocrinol Metab 2005; 90: 2110-2116.

18. Amar L, Bertherat J, Baudin E, et al. Genetic testing in pheochromocytoma or functional paraganglioma. J Clin Oncol 2005; 23: 8812-8818.

19. Karasek D, Frysak Z, Pacak K. Genetic testing for pheochromocytoma. Curr Hypertens Rep 2010; 12: 456-464. 